\title{
Novel tests for diagnosing tuberculous pleural effusion: what works and what does not?
}

\author{
A. Trajman*, M. Pai ${ }^{\#}$, K. Dheda ${ }^{\top,+}$, R. van Zyl Smit ${ }^{\top,+}$, A.A. Zwerling ${ }^{\#}$, R. Joshi ${ }^{\S}$, \\ S. Kalantri ${ }^{\S}$, P. Daley ${ }^{\dagger}$ and D. Menzies ${ }^{\#}$
}

\begin{abstract}
Tuberculous pleuritis is a common manifestation of extrapulmonary tuberculosis and is the most common cause of pleural effusion in many countries. Conventional diagnostic tests, such as microscopic examination of the pleural fluid, biochemical tests, culture of pleural fluid, sputum or pleural tissue, and histopathological examination of pleural tissue, have known limitations. Due to these limitations, newer and more rapid diagnostic tests have been evaluated. In this review, the authors provide an overview of the performance of new diagnostic tests, including markers of specific and nonspecific immune response, nucleic acid amplification and detection, and predictive models based on combinations of markers. Directions for future development and evaluation of novel assays and biomarkers for pleural tuberculosis are also suggested.
\end{abstract}

KEYWORDS: Biomarkers, diagnosis, pleuritis, sensitivity, specificity, tuberculosis

$\mathbf{T}$ uberculous pleuritis is a common manifestation of extrapulmonary tuberculosis (TB) and is the most common cause of pleural effusion in many countries [1-3]. Pleural TB occurs as a result of a TB antigen entering the pleural space, usually through the rupture of a subpleural focus, followed by a local, delayed hypersensitivity reaction mediated by $\mathrm{CD} 4+$ cells [4]. This process may occur during primary or reactivation TB and may or may not involve viable bacilli entering the pleural space [5].

The presence of mycobacterial antigens in the pleural space elicits an intense immune response, initially by neutrophils and macrophages $[6,7]$, followed by interferon (IFN)- $\gamma$-producing T-helper cell (Th) type 1 lymphocytes [4, 8], resulting in a lymphocyte-predominant exudative effusion. This cellular trafficking is facilitated by homing surface markers and chemokine gradients [9, 10]. This intense but poorly understood local immune response by sensitised lymphocytes to TB antigen is synonymous with the Koch phenomenon [11]. A recent study indicates that cells of an alternative T-cell profile, CD4(+)/CD25(+)/FoxP3(+) regulatory T-cells, are expanded in TB pleural effusion and can suppress some effector responses, although the precise role of these cells remains unclear.
Conventional diagnostic tests for pleural TB include microscopic examination of the pleural fluid for acid-fast bacilli, mycobacterial culture of pleural fluid, sputum or pleural tissue, and histopathological examination of pleural tissue for granulomatous inflammation. These tests have recognised limitations for clinical use, although, in combination, they have been recognised as the best reference standard for evaluation of the accuracy of novel tests [12].

Microscopy of the pleural fluid for acid-fast bacilli is positive in $<5 \%$ of TB pleuritis cases, due to the paucibacillary nature of the disease $[12,13]$. Mycobacterial culture of pleural fluid also has low sensitivity $(24-58 \%)[13,14]$ and is limited by the lengthy delay in obtaining results: up to 8 weeks if solid culture media are used. Biopsy of pleural tissue for combined histological examination and mycobacterial culture of pleural fluid and tissue is the most sensitive of the currently available diagnostic methods, but may still be falsely negative in $15-20 \%$ of these cases $[12,15]$. In addition, pleural biopsy is invasive, and yield as well as complication rates are dependent on the skill of the operator because it is technically difficult, particularly in children. Hence, biopsy adds considerable risk and cost to

\section{AFFILIATIONS}

*Pró-reitoria de Saúde, Gama Filho University, Rio de Janeiro, Brazil.

\#Montreal Chest Institute, McGill University, Montreal, QC, Canada. "Division of Pulmonology, Dept of Medicine, University of Cape Town Rondebosch, South Africa.

${ }^{+}$Centre for Infectious Diseases and International Health, Royal Free and UCL Medical School, London, UK.

${ }^{5}$ Dept of Medicine, Mahatma Gandhi Institute of Medical Sciences,

Sevagram, and

${ }^{f}$ Dept of Medicine, Christian Medical College, Vellore, India.

\section{CORRESPONDENCE}

M. Pai

Dept of Epidemiology

Biostatistics and Occupational Health

McGill University

1020 Pine Avenue West

Montreal

QC

Canada H3A 1 A2

Fax: 15143984503

E-mail: madhukar.pai@mcgill.ca

Received:

November 062007

Accepted after revision:

January 242008

SUPPORT STATEMENT

For a full list of all grants and funding received, please see the

Acknowledgements section.

STATEMENT OF INTEREST

None declared.

European Respiratory Journal

Print ISSN 0903-1936

Online ISSN 1399-3003 
the workup. Mycobacterial culture of spontaneous sputum or gastric lavage has a variable yield, from $0 \%$ [16] to 30\% [17], depending on the presence of associated lung parenchymal lesions [17]. Since patients with pleural TB rarely produce sputum spontaneously, routine collection of induced sputum has been proposed for the diagnosis of pleural TB. In a prospective study of 113 patients with confirmed pleural TB, induced sputum had a sensitivity of $52 \%$, compared with $12 \%$ sensitivity of pleural fluid culture [18]. Due to these limitations of conventional tests, plus the delay of several weeks for mycobacterial culture results, newer rapid tests and biomarkers have been evaluated. The present review provides a narrative overview of the literature on the tests, summarised in table 1 and illustrated in figure 1 , and reviews their performance characteristics for the diagnosis of pleural TB.

For clarity, all tests were grouped into the following categories, although some overlap exists: 1) nonspecific inflammatory and immune response markers; 2) specific markers of immune response; 3) nucleic acid amplification tests; and 4) scoring systems based on combinations of tests.

\section{NONSPECIFIC INFLAMMATORY AND IMMUNE RESPONSE MARKERS \\ Adenosine deaminase}

Adenosine deaminase (ADA), released by activated lymphocytes, macrophages and neutrophils, is a nonspecific marker of inflammation. The ADA2 isoenzyme released from monocytes and macrophages is the predominant contributor to total ADA activity [49]. A high diagnostic accuracy of ADA activity measurement has been reported in several studies. In a metaanalysis, GRECO et al. [19] found that among 31 studies published prior to 2000 , including 4,738 patients, the pooled sensitivity was $92 \%$ (range $56-100 \%$ ) and pooled specificity was $89 \%(55-100 \%)$ using composite reference standards including culture, histology, sputum culture and response to therapy. The specificity for discriminating TB from malignant effusions, an important differential diagnosis in elderly patients, remained high $(95 \%)$ but was disappointingly low for parapneumonic effusions.

GRECO et al. [19] concluded that in low and intermediate TB incidence settings, the negative predictive value was sufficient that a negative ADA activity result would preclude the need for pleural biopsy. However, in the same settings, the positive predictive value was poor. In contrast, in high prevalence settings, a positive ADA would provide a 99\% post-test probability of TB. This crucial point, that the predictive value of a test is highly dependent on the prevalence of the disease, explains the differences in performance of this test in different studies. Thus, the clinical context must be taken into account when interpreting the ADA result.

Many studies in the review by GRECO et al. [19] included $<30$ patients, resulting in wide confidence intervals (CIs) around sensitivity and specificity estimates and significant heterogeneity

TABLE 1 Novel tests and biomarkers for the diagnosis of tuberculous pleural effusions

Test or biomarker

Performance characteristics

[Ref.]

\section{Nonspecific inflammatory and immune response markers \\ ADA activity}

Neopterin

Leptin

Lysozyme

Fibronectin

IFN- $\gamma$ measurement

IL-2

TNF- $\alpha$, IL-1 $\beta$

Complement activation

CD4+ T-cell count

Specific markers of immune response

T-cell response to ESAT-6 and CFP-10

Serologic (antibody) tests

\section{Detection of Mycobacterium tuberculosis} nucleic acid by commercial NAATs
Consistent high sensitivity and reasonable specificity

Inexpensive, simple, rapid

Best performance among nonspecific inflammatory markers

Limited data, variable sensitivity and specificity

Limited data

Low specificity

Limited data, comparison with malignant effusion only, no established cut-off

Consistent high sensitivity and specificity, kit available, expensive

$[19,31,32]$

Best performance of the group

Lower sensitivity and specificity than IFN- $\gamma$

Limited data, no established cut-off

Intermediate sensitivity and specificity, better performance in diagnosis of rheumatic

diseases and parapneumonic effusions

Limited value for TB diagnosis

Limited data, few patients, specificity data needed

Very low sensitivity, high specificity, best sensitivity with an in-house method detecting

antibodies against MPT-64 and MT-10.3

High specificity with TBGL antigen

Other antigens (P32, A60) disappointing

Low and variable sensitivity, high specificity, kits not approved for extrapulmonary specimens

ADA: adenosine deaminase; IFN: interferon; IL: interleukin; TNF: tumour necrosis factor; ESAT: early secreted antigenic target; CFP: culture filtrate protein; NAAT: nucleic acid amplification test; TB: tuberculosis; TBGL: tuberculous glycolipid. 


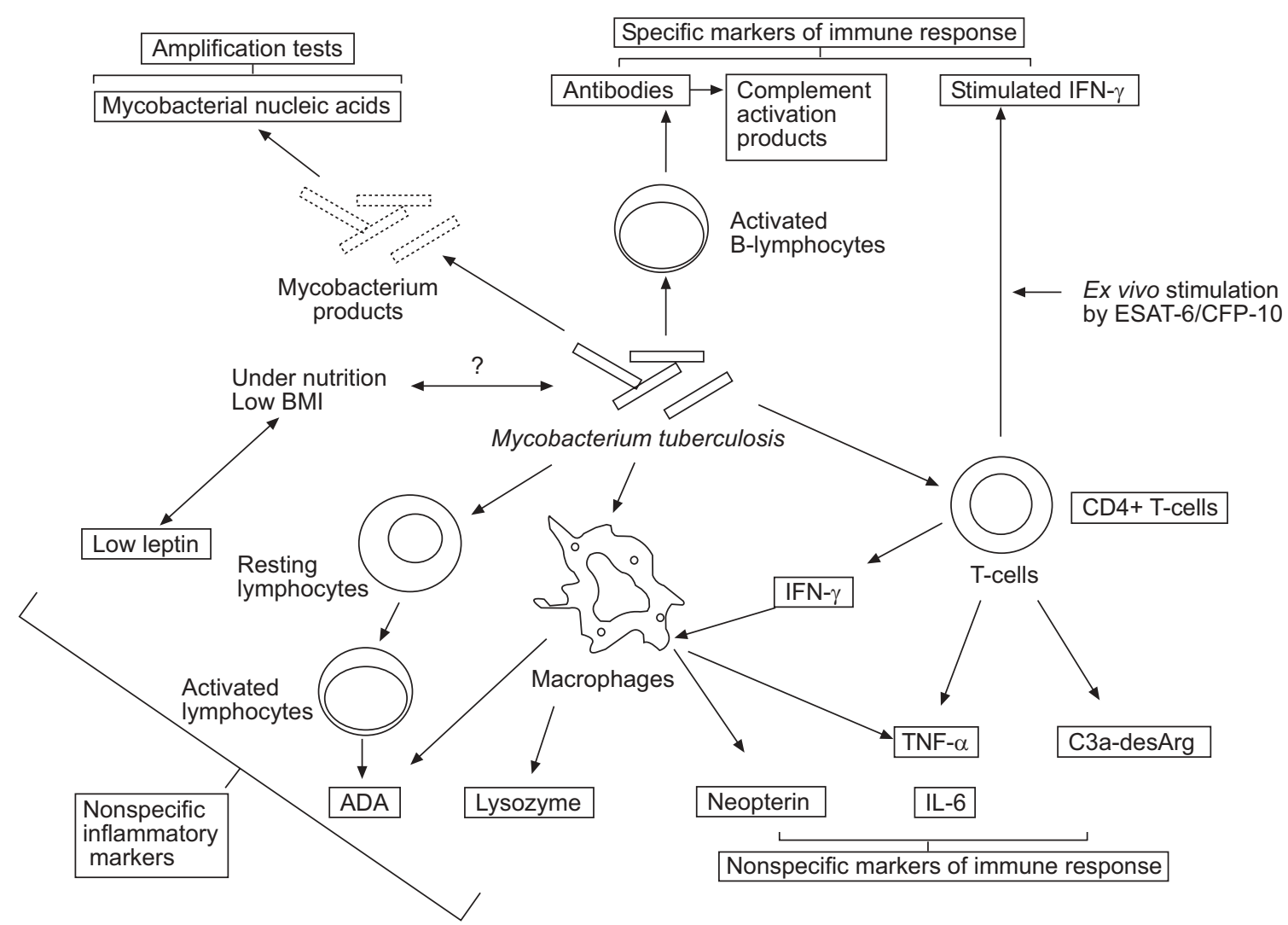

FIGURE 1. Schematic representation of pathways and systems involved in biomarkers for pleural tuberculosis. IFN: interferon; ESAT: early secreted antigenic target; CFP: culture filtrate protein; BMI: body mass index; ADA: adenosine deaminase; TNF: tumour necrosis factor; IL: interleukin.

between estimates. In addition, few studies reported blinding or described in detail the inclusion criteria for the control group.

Using histopathology as the reference standard, pleural fluid ADA activity, PCR and immunoglobulin (Ig)A-ELISA tests were evaluated in a high TB incidence country [20] among 77 patients with pleural effusion, 60 of whom had TB pleuritis. ADA activity was the only test that had a significantly higher sensitivity than histopathological examination. The study included only 17 nontuberculous effusions, and, therefore, specificity could not be adequately estimated [21].

Determination of ADA isoenzyme activity in pleural fluid may increase the accuracy of the test. ADA1 is secreted by lymphocytes and monocytes, while ADA2 is secreted only by monocytes and is found in a higher concentration in $\mathrm{TB}$ pleuritis [22, 23]. However, because the additional yield is small, a study would require a very large sample size to demonstrate that isoenzymes have significantly higher specificity than total ADA activity.

\section{Neopterin}

Neopterin is a marker of Th1 immune activation, as it is secreted by activated macrophages. Neopterin levels in pleural fluid have been found to be higher in patients with TB than patients with malignancy [24, 25] or other [26] conditions. Very high levels of neopterin have been observed in uremic pleural effusions [27], raising doubts about its specificity for TB. In one study that used histological evidence of caseating granuloma as the reference standard for TB, the sensitivity and specificity of neopterin were 44 and $85 \%$, respectively [25]. In a second study, which used ADA as the reference standard, the sensitivity was $85 \%$ and the specificity was $93 \%$ [26]. However, ADA may not be a suitable reference standard because it is also an indicator of nonspecific inflammation, so a high degree of correlation may reflect the same underlying inflammatory response as neopterin.

\section{Leptin}

Leptin, a $16-\mathrm{kDa}$ product of the obese (ob) gene, may be involved in cross-regulation between nutritional status and the immune response in TB. Serum leptin levels have been shown to be reduced in patients with active pulmonary TB [50] and cancer [51]. One study evaluated total leptin pleural fluid levels and leptin pleural fluid/serum ratio in 17 patients with pleural TB, and parapneumonic $(n=7)$ and malignant $(n=21)$ effusions [28]. The reference standard for TB in this study was the presence of granuloma in pleural tissue. ADA activity was also measured in pleural fluid. Pleural ADA activity sensitivity and specificity were both $100 \%$ and pleural leptin $\left(<9.85 \mathrm{ng} \cdot \mathrm{mL}^{-1}\right)$ sensitivity and specificity were $82 \%(p=0.01)$. Thus, there is no evidence that leptin performs better than pleural fluid ADA.

\section{Lysozyme}

Lysozyme is present in epithelial cells of granulomas, macrophages and activated granulocytes. In a study of patients with pleural effusion in a setting with high TB incidence, VALDÉs et al. [29] measured ADA activity in 405 specimens, lysozyme in 
276 specimens and IFN- $\gamma$ in 145 specimens. Using culture of pleural fluid or tissue and histopathological evidence of caseous granuloma in pleural tissue as the reference standard, the sensitivity of ADA, lysozyme and IFN- $\gamma$ were 100, 85.7 and $94.2 \%$, respectively, while specificities were 94.9, 61.6 and $91.8 \%$, respectively. Thus, ADA and IFN- $\gamma$ were more accurate than lysozyme levels. However, 51 patients were excluded from the analysis because a final definitive diagnosis could not be achieved, and the criteria for selection of specimens for lysozyme and IFN- $\gamma$ testing were not reported. For these reasons, potential selection bias cannot be excluded.

MORIWAKI et al. [30] found that lysozyme could discriminate malignant from tuberculous effusions with a sensitivity of $100 \%$ and a specificity of $83 \%$, compared with culture and/or histopathology of pleural tissue. The pleural fluid/serum lysozyme ratio had a sensitivity of $100 \%$ and a specificity of $88 \%$. The mean fibronectin concentration in pleural fluid with tuberculous effusion was significantly higher than that in malignant effusion, but there was a marked overlap between the groups. In this study, lysozyme and fibronectin were less accurate than pleural fluid ADA [30].

\section{Cytokines}

Of all the cytokines, IFN- $\gamma$ has been the most studied. The evidence for use of IFN- $\gamma$ in pleural fluid for diagnosis of TB has been reviewed by GRECO et al. [19] and JIANG et al. [31]. In the latter review, based on 22 studies including 782 TB patients and 1,319 non-TB patients, the summary estimate of sensitivity was $89 \%$ (95\% CI $87-91 \%$ ) and of specificity was $97 \%$ (96-98\%) [31]. IFN- $\gamma$ was more sensitive and specific for the diagnosis of pleural TB than interleukin (IL)-12p40, IL-18, immunosuppressive acidic protein or soluble IL-2 receptor, in a study that directly compared these tests in the same samples [33]. In another study, the accuracy of IFN- $\gamma$ was superior to that of ADA [19].

The concentration of other pro-inflammatory cytokines, such as tumour necrosis factor- $\alpha$ and IL- $1 \beta$, has been shown to be higher in tuberculous than in malignant effusions, but no cutoff value for positivity has been established [34]. In another study, IL- 8 and IL-6, and soluble IL-6 receptor were detected in carcinomatous but not in tuberculous pleural effusions [52].

\section{Complement activation}

PORCEL et al. [53] measured SC5b-9 and C3a-desArg, two products of complement activation, in the pleural fluid of 83 patients using commercial ELISA tests. The sensitivity was $84 \%$ for SC5b-9 and $81 \%$ for C3a-desArg. HARA et al. [35] showed SC5b-9 had a higher sensitivity (100\%) and ADA activity had a higher specificity than conventional tests (culture and/or histopathological examination). Discrimination of TB pleuritis from malignant effusions was better than discrimination from autoimmune or parapneumonic effusions. In fact, the SC5b-9 level seemed more useful for the diagnosis of rheumatoid pleural effusions [36] and for differentiation of empyema from uncomplicated parapneumonic effusions [37].

\section{Cell subsets}

Tuberculous effusions are usually rich in mononuclear cells [12]. In one study, although pleural fluid ADA activity correlated with the number of CD4+ T-cells in the pleural space [38], the number of pleural CD4+ cells was not an accurate diagnostic test for TB.

In conclusion, among the nonspecific inflammatory biomarkers that have been evaluated, ADA and IFN- $\gamma$ are the most accurate for pleural TB diagnosis, with consistently high sensitivity in many studies. Specificity of ADA is a concern because of the nonspecific nature of inflammatory response. Most studies used in-house methods for ADA activity estimation, which may demonstrate considerable variability in performance. Commercial kits have been used in a few studies, but these kits should be validated in different settings. ADA activity measurement is simple and inexpensive to perform and does not require special equipment, making it an attractive option for settings with high $\mathrm{TB}$ incidence and limited resources. In contrast, measurement of IFN- $\gamma$ is expensive, estimated to be as much as the cost of treatment for six TB patients [32].

\section{SPECIFIC MARKERS OF IMMUNE RESPONSE $T$-cell response to specific antigens}

Recently, in vitro, T-cell-based IFN- $\gamma$ release assays (IGRAs) have been developed and licensed for diagnosis of latent TB infection. Normally, these tests use peripheral blood mononuclear cells (PBMCs), but they can be used with pleural fluid mononuclear cells. These assays detect IFN- $\gamma$ secreted by mononuclear cells in response to in vitro stimulation with the Mycobacterium tuberculosis-specific antigens early secreted antigenic target- 6 and culture filtrate protein-10. The genes that encode these antigens are not present in any of the M. bovis bacille Calmette-Guerin (BCG) strains or certain common nontuberculous (environmental) mycobacteria [54]. Thus, in theory, the test should not cross-react with antigens present due to BCG vaccination [54,55]. In a recent study [39], the T-SPOT.TB test (Oxford Immunotec Ltd, Oxford, UK) was performed on PBMCs and mononuclear cells from pleural fluid from 20 patients clinically suspected to have TB pleuritis and 21 subjects with other diagnoses. The sensitivity of T-SPOT.TB was $90 \%$ using the blood assay and $95 \%$ for pleural fluid, but the specificity was only $67 \%$ for blood and $76 \%$ for pleural fluid. This poor specificity may reflect positive reactions due to coincidental latent TB infection, coexisting or transient infection.

An alternative commercially available T-cell assay using an ELISA platform is the QuantiFERON ${ }_{\mathbb{B}}$-TB Gold in-tube test (Cellestis Ltd, Carnegie, Australia), which is more amenable to high throughput and flexibility with analysis. However, unpublished preliminary observations indicate that high background IFN- $\gamma$ levels in the negative control tube precludes the use of ex vivo unprocessed pleural fluid in the assay. Prespecified numbers of mononuclear cells in culture medium may overcome this drawback.

IGRAs, designed to detect latent TB infection, will only be useful for the diagnosis of pleural TB disease if it can be shown that persons with latent TB infection and pleural effusions due to nontuberculous causes, such as cancer, have positive IGRA using serum, but negative IGRA using pleural fluid. This finding would suggest that TB-sensitised lymphocytes remain in peripheral blood but are not present among the lymphocytes found in the pleural fluid. This hypothesis as yet remains 
unproven. Until this issue is settled, these assays may not offer any additional value for the diagnosis of pleural effusion, beyond the measurement of free IFN- $\gamma$ in the pleural fluid.

\section{B-cell response (antibody detection)}

Although detection of serum antibodies against TB antigens is known to have poor and highly variable sensitivity and specificity [56, 57], attempts have been made to detect antibodies in pleural fluid specimens using techniques such as ELISA. Among pleural fluid antibody tests, detection of IgA against MPT-64 and MT-10.3 (Rv3019c), two recombinant protein antigens, had the best sensitivity [20,40]. Anti-A60 IgM and IgG antibodies in pleural fluid had suboptimal sensitivity for TB [41]. Using antibodies against five different antigens, CHIERAKUl et al. [42] found the performance of serology of pleural fluid to be disappointing, with sensitivity and specificity $<60 \%$. Anti-P32 levels were reported to be higher among five patients with pleural TB, but the sensitivity and specificity were not reported [43]. Sensitivity to anti-lipoarabinomannan (LAM) and to anti-TB glycolipid antibodies was very low in other studies, although specificity was high [44-47].

One study reported a simple and cost-effective diagnostic tool (an in-house TB screen test) for pulmonary and extrapulmonary $\mathrm{TB}$, using a liposome agglutination assay to detect serological responses to purified mycobacterial glycolipid antigens [58]. The assay was able to detect very low antiglycolipid antibody concentrations in the serum of individuals with TB. The status for latent TB in the control group was not reported. To date, the test has not been applied to pleural fluid specimens, although four subjects with pleural TB were serum tested [58].

In conclusion, serologic tests using antibodies against mycobacterial protein and glycolipids show some potential for the diagnosis of pleural TB because of their high specificity, but are limited by very poor sensitivity.

\section{DETECTION OF M. TUBERCULOSIS NUCLEIC ACID SEQUENCES BY AMPLIFICATION TESTS}

Several commercial and in-house assays exist for the amplification and detection of $M$. tuberculosis nucleic acids from specimens such as sputum. These tests have also been used with specimens such as pleural fluids. In a meta-analysis of 40 studies of nucleic acid amplification tests (NAATs) for pleural TB, PAI et al. [48] reported that commercial NAATs have a potential role in confirming TB pleuritis because of high specificity (98 (95\% CI 96-98)\%). However, these tests had low and variable sensitivity (62 (43-77)\%) and, therefore, were not useful in excluding the disease. The accuracy of in-house NAATs was poorly defined because of heterogeneity across studies, presumably reflecting the heterogeneity of in-house test protocols. Similar results have been reported with NAATs for TB meningitis [59] and TB lymphadenitis [60].

The reasons for the low sensitivity of NAATs in pleural fluid specimens are unclear. The presence of inhibitory substances in pleural fluid is an unsatisfactory explanation, as most commercial assays have an internal positive control to account for inhibition. The small amount of mycobacteria in pleural effusion may play some role, but the low sensitivity is more likely to be related to a technical aspect of nucleic acid extraction. Thus, caution is needed when interpreting negative NAAT results in pleural fluids, and performance will depend largely on pre-test probability.

In conclusion, the evidence is consistent that NAATs have modest sensitivity but excellent specificity for pulmonary and extrapulmonary TB. At present, no commercial kit has been approved by the US Food and Drug Administration for the diagnosis of extrapulmonary TB, and NAATs cannot be used in isolation to rule in or rule out pleural TB.

\section{SCORING SYSTEMS BASED ON COMBINATIONS OF MARKERS}

Due to the limitations of individual tests, scoring systems have been developed, based on the results of multiple tests (table 2). A scoring system that makes use of simple clinical and pleural fluid data was created by PORCEL and VIVES [61] to discriminate between TB and malignant pleural effusions. Two models were developed, one with and one without ADA activity measurement. In the first model, four variables predicted a tuberculous aetiology: $\mathrm{ADA} \geqslant 40 \mathrm{U} \cdot \mathrm{L}^{-1}$ (five points); age $<35 \mathrm{yrs}$ (two points); temperature $\geqslant 37.8^{\circ} \mathrm{C}$ (two points); and pleural fluid red blood cell count $<5 \times 10^{9} \cdot \mathrm{L}^{-1}$ (one point). In the second model, TB was predicted by: age $<35$ yrs (two points); temperature $\geqslant 37.8^{\circ} \mathrm{C}$ (two points); pleural fluid red blood cell count $<5 \times 10^{9} \cdot \mathrm{L}^{-1}$ plus no history of malignancy (three points); pleural protein $\geqslant 50 \mathrm{~g} \cdot \mathrm{L}^{-1}$ (one point); and pleural fluid/serum lactate dehydrogenase ratio $\geqslant 2.2$ (one point). Summated scores of at least five in model one and at least six in model two yielded measures of sensitivity (95 and $97 \%$, respectively) and specificity (94 and 91\%, respectively) for discriminating TB from malignant effusions.

VILLEGAS et al. [62] also proposed a combination of clinical and biological markers. In their study, ADA activity, IFN- $\gamma$ levels

\section{TABLE 2 Combination of tests and biomarkers for the diagnosis of tuberculous pleural effusions}

\begin{tabular}{|c|c|c|}
\hline Combinations of markers & Reported accuracy & [Ref.] \\
\hline Age + fever + red blood cells + ADA & Very high sensitivity, high specificity & [61] \\
\hline $\begin{array}{l}\text { Duration of symptoms + protein + leukocyte count + } \\
\text { lymphocytes } \%+\text { ADA }\end{array}$ & High sensitivity and specificity & [63] \\
\hline ADA + lymphocyte/neutrophil ratio & High sensitivity and specificity & [64] \\
\hline
\end{tabular}


and PCR were tested in the same samples for the diagnosis of microbiologically and/or histologically confirmed pleural TB. The sensitivities of ADA activity, IFN- $\gamma$ levels and PCR were 88,86 and $74 \%$, respectively, and the specificities were 86,97 and $90 \%$, respectively. The combination of PCR, IFN- $\gamma$ and ADA activity allowed an increase of sensitivity and specificity compared with individual methods in isolation. However, in other studies using different tests in the same samples, in which ADA activity had a higher accuracy, no other test significantly added sensitivity to the ADA $[20,65]$.

In a logistic regression model, a combination of duration of disease, protein levels, total leukocyte count, percentage of lymphocytes and ADA activity measurement was $>95 \%$ sensitive and specific for the diagnosis of $\mathrm{TB}$ in a high prevalence setting [63]. The first four variables added specificity to ADA activity, without loss of sensitivity. The lymphocyte/neutrophil ratio has also added to ADA specificity in another study of 472 pleural effusions [64].

More models, which take into account clinical features and simple, inexpensive and rapid diagnostic tests, can be developed with the use of modelling tools. These models are highly relevant to clinicians practising in poor resource settings. Even when ADA is unavailable, use of demographic and clinical information in combination with a cell differential count, in high incidence settings, can yield a diagnosis of TB with a high predictive value.

\section{EMERGING TECHNOLOGIES THAT NEED TO BE EVALUATED FOR PLEURAL TB}

In the past few years, several new TB diagnostics have been developed. These include tests such as loop-mediated isothermal amplification, detection of LAM (a TB antigen) using ELISA, phage-based assays and rapid culture systems [66, 67]. To date, none of these have been adequately evaluated for TB pleuritis.

In addition to laboratory tests, biological processes found in nature are being used to inspire the development of new technologies. Artificial intelligence represents an attempt to simulate the human brain in resolving complex questions by breaking them down into small problems. Neural network systems have been used to extract relevant information from complex databases, categorise them into groups with similar characteristics and organise them into distinct patterns [68]. The neural network system can help in identifying patients' variables that may not make biomedical sense but could be relevant for the diagnosis of diseases. It can also detect errors in databases. This tool has been useful in predicting pulmonary TB in patients with negative sputum smears $[69,70]$. The tool can also easily generate scoring models based on clinical and laboratory data. Research is needed to evaluate the usefulness of neural network systems for the diagnosis of pleural TB.

\section{FUTURE DIRECTIONS}

Table 3 presents some suggested research directions. Diagnostic studies on pleural TB are particularly needed in specific populations, including individuals with HIV infection, children and other high-risk populations where traditional diagnostic tests have worse yield. In addition, commercially available tests for diagnosis of pulmonary TB should be validated for diagnosis of pleural TB. Better tests are needed, and/or available tests, such as NAAT or serologic tests that have good specificity, need to be modified to improve sensitivity.

Multidrug-resistant and extensively drug-resistant TB are spreading worldwide and have become a special issue for $\mathrm{TB}$ control. There are no reports on the drug-resistance profile of strains isolated from pleural specimens, but given the trend of ever-increasing resistance, development of new techniques, such as NAATs, to identify drug-resistant strains causing pleural TB will be important.

Efforts are also needed to improve the quality and reporting of TB diagnostic studies, according to the Standards for Reporting of Diagnostic Accuracy recommendations [71]. The main limitation of many published studies is the absence of a welldefined reference standard (culture and histopathological evidence of TB), leading to test performance results that may be biased. In addition to the lack of a definitive gold standard, diagnostic studies are plagued by methodological and design flaws that compromise validity, such as lack of a consecutive or

\section{TABLE 3 Recommendations for future research}

Test or biomarker

Nonspecific inflammatory and immune response markers

Specific markers of immune response T-cell response

B-cell response (antibodies)

Detection of Mycobacterium tuberculosis

nucleic acid by amplification tests

Combinations of markers
Research needs and recommendations

Commercial kits for ADA need to be validated in large studies with well-defined reference standards. The roles of cytokines and biological markers other than IFN- $\gamma$ need to be established, which may require genomic or proteomic approaches.

Potentially should provide high specificity; further studies, particularly in high-incidence settings, required. Improvements needed in specificity and sensitivity of IGRAs, and DNA extraction methods. Antigens other than ESAT-6 and CFP-10 need to be tested.

Antibodies against glycolipid antigens need to be developed and tested. Antibodies to protein antigens need sensitivity improvement, and more immunogenic antigens need to be found.

Potentially should provide high specificity; need sensitivity improvement. More commercial tests need to be developed. Existing kits need to be validated in pleural fluid. Potential to provide drug sensitivity profile.

More predictive models need to be developed using different clinical and laboratory data, with aid of informatics; artificial intelligence.

ADA: adenosine deaminase; IFN: interferon; IGRA: IFN- $\gamma$ release assay; ESAT: early-secreted antigenic target; CFP: culture filtrate protein. 
random patient-sampling method, use of a case-control design (where severe cases are often compared with healthy controls) and lack of blinding [72-74]. Statistical analysis of results should receive special attention in future studies [21, 75]. Sensitivities and specificities are proportions, and their confidence intervals must be calculated and reported [75]. When using statistical tests for comparison of sensitivities, only patients with the disease should be compared, while for specificity, only patients without the disease should be compared [21].

\section{CONCLUSION}

Diagnosis of pleural TB remains a challenge, as summarised in table 1. Among nonconventional tests, ADA and IFN- $\gamma$ have the best sensitivity and specificity, but they are biomarkers of the inflammatory process in the pleural space and do not confirm the aetiologic agent. NAATs and serology are promising but need further development to improve sensitivity. Limited evidence is available for other novel tests and biomarkers.

Combinations of tests seem to perform better than any single test, especially combinations that include adenosine deaminase, but only a few studies have evaluated scoring systems (table 2). Further work is necessary to identify the best (and simplest) combination that will be most useful in clinical practice.

\section{ACKNOWLEDGEMENTS}

A. Trajman received funding from an International Clinical, Operational and Health Services Research Training Award for AIDS and TB, Fogarty International Center, National Institutes of Health, no. 5U2 R TW006883-03. M. Pai is supported by a Canadian Institutes of Health Research New Investigator Award, and D. Menzies by a Fonds de la Recherche en Santé du Québec Chercheur National salary award. K. Dheda is supported by a South African National Research Foundation Research Chair award and a Medical Research Council career development award. $\mathrm{R}$. Joshi is a recipient of a training fellowship from the Fogarty AIDS International Training Program, grant no. 1-D43-TW00003-17, USA.

\section{REFERENCES}

1 Liam CK, Lim KH, Wong CM. Causes of pleural exudates in a region with a high incidence of tuberculosis. Respirology 2000; 5: 33-38.

2 Selig L, Cunha AJLA, Teixeira EG, Belo MTCT, Branco MMC, Trajman A. [Anti-HIV testing for tuberculosis patients in Rio de Janeiro State]. Pulmão RJ 2001; 10: 8-13.

3 Valdés L, Pose A, San José E, Martinez Vázquez JM. Tuberculous pleural effusions. Eur J Intern Med 2003; 14: 77-88.

4 Barnes PF, Mistry SD, Cooper CL, Pirmez C, Rea TH, Modlin RL. Compartmentalization of a CD4+ T lymphocyte subpopulation in tuberculous pleuritis. J Immunol 1989; 142: 1114-1119.

5 Kim HJ, Lee HJ, Kwon SY, et al. The prevalence of pulmonary parenchymal tuberculosis in patients with tuberculous pleuritis. Chest 2006; 129: 1253-1258.

6 Antony VB, Repine JE, Harada RN, Good JT Jr, Sahn SA. Inflammatory responses in experimental tuberculosis pleurisy. Acta Cytol 1983; 27: 355-361.
7 Antony VB, Sahn SA, Antony AC, Repine JE. Bacillus Calmette-Guérin-stimulated neutrophils release chemotaxins for monocytes in rabbit pleural spaces and in vitro. $J$ Clin Invest 1985; 76: 1514-1521.

8 Sharma SK, Mitra DK, Balamurugan A, Pandey RM, Mehra NK. Cytokine polarization in miliary and pleural tuberculosis. J Clin Immunol 2002; 22: 345-352.

9 Gopi A, Madhavan SM, Sharma SK, Sahn SA. Diagnosis and treatment of tuberculous pleural effusion in 2006. Chest 2007; 131: 880-889.

10 Mitra DK, Sharma SK, Dinda AK, Bindra MS, Madan B, Ghosh B. Polarized helper T cells in tubercular pleural effusion: phenotypic identity and selective recruitment. Eur J Immunol 2005; 35: 2367-2375.

11 Dheda K, Booth H, Huggett JF, Johnson MA, Zumla A, Rook GA. Lung remodeling in pulmonary tuberculosis. $J$ Infect Dis 2005; 192: 1201-1209.

12 Light RW. Useful tests on the pleural fluid in the management of patients with pleural effusions. Curr Opin Pulm Med 1999; 5: 245-249.

13 Bueno EC, Clemente GM, Castro CB, et al. Cytologic and bacteriologic analysis of fluid and pleural biopsy specimens with Cope's needle. Study of 414 patients. Arch Intern Med 1990; 150: 1190-1194.

14 Seibert AF, Haynes J Jr, Middleton R, Bass JB Jr. Tuberculous pleural effusion. Twenty-year experience. Chest 1991; 99: 883-886.

15 Palomino JC, Leão SC, Ritacco V, eds. Tuberculosis 2007. From Basic Science to Patient Care. 1st Edn. www. tuberculosistextbook.com/tuberculosis2007.pdf Date last accessed: February 21, 2008. Date last updated: June 2007.

16 Arrington CW, Hawkins JA, Richert JH, Hopeman AR. Management of undiagnosed pleural effusions in positive tuberculin reactors. Am Rev Respir Dis 1966; 93: 587-593.

17 Berger HW, Mejia E. Tuberculous pleurisy. Chest 1973; 63: 88-92.

18 Conde MB, Loivos AC, Rezende VM, et al. Yield of sputum induction in the diagnosis of pleural tuberculosis. Am J Respir Crit Care Med 2003; 167: 723-725.

19 Greco S, Girardi E, Masciangelo R, Capoccetta GB, Saltini C. Adenosine deaminase and interferon- $\gamma$ measurements for the diagnosis of tuberculous pleurisy: a metaanalysis. Int J Tuberc Lung Dis 2003; 7: 777-786.

20 Trajman A, Kaisermann C, Luiz RR, et al. Pleural fluid ADA, IgA-ELISA and PCR sensitivities for the diagnosis of pleural tuberculosis. Scand J Clin Lab Invest 2007; 67: 877-884.

21 Trajman A, Luiz RR. McNemar Chi(2) test revisited: comparing sensitivity and specificity of diagnostic examinations. Scand J Lab Clin Invest 2008; 68: 77-80.

22 Pérez-Rodríguez E, Pérez Walton IJ, Sanchez Hernández JJ, et al. ADA1/ADAp ratio in pleural tuberculosis: an excellent diagnostic parameter in pleural fluid. Respir Med 1999; 93: 816-821.

23 Gorguner M, Cerci M, Gorguner I. Determination of adenosine deaminase activity and its isoenzymes for diagnosis of pleural effusions. Respirology 2000; 5: 321-324.

24 Baganha MF, Mota-Pinto A, Pêgo MA, Marques MA, Rosa MA, Cordeiro AJ. Neopterin in tuberculous and neoplastic pleural fluids. Lung 1992; 170: 155-161.

25 Cok G, Parildar Z, Basol G, et al. Pleural fluid neopterin levels in tuberculous pleurisy. Clin Biochem 2007; 40: 876-880. 
26 Tozkoparan E, Deniz O, Cakir E, et al. The diagnostic values of serum, pleural fluid and urine neopterin measurements in tuberculous pleurisy. Int J Tuberc Lung Dis 2005; 9: 1040-1045.

27 Chiang CS, Chiang CD, Lin JW, Huang PL, Chu JJ. Neopterin, soluble interleukin-2 receptor and adenosine deaminase levels in pleural effusions. Respiration 1994; 61: 150-154.

28 Celik G, Kaya A, Poyraz B, et al. Diagnostic value of leptin in tuberculous pleural effusions. Int J Clin Pract 2006; 60: 1437-1442.

29 Valdés L, San José E, Alvarez D, et al. Diagnosis of tuberculous pleurisy using the biologic parameters adenosine deaminase, lysozyme, and interferon- $\gamma$. Chest 1993; 103: 458-465.

30 Moriwaki Y, Kohjiro N, Itoh M, et al. Discrimination of tuberculous from carcinomatous pleural effusion by biochemical markers: adenosine deaminase, lysozyme, fibronectin and carcinoembryonic antigen. Jpn J Med 1989; 28: 478-484.

31 Jiang J, Shi HZ, Liang QL, Qin SM, Qin XJ. Diagnostic value of interferon- $\gamma$ in tuberculous pleurisy: a metaanalysis. Chest 2007; 131: 1133-1141.

32 Sharma SK, Banga A. Pleural fluid interferon- $\gamma$ and adenosine deaminase levels in tuberculosis pleural effusion: a cost-effectiveness analysis. J Clin Lab Anal 2005; 19: 40-46.

33 Hiraki A, Aoe K, Eda R, et al. Comparison of six biological markers for the diagnosis of tuberculous pleuritis. Chest 2004; 125: 987-989.

34 Hua CC, Chang LC, Chen YC, Chang SC. Proinflammatory cytokines and fibrinolytic enzymes in tuberculous and malignant pleural effusions. Chest 1999; 116: 1292-1296.

35 Hara N, Abe M, Inuzuka S, Kawarada Y, Shigematsu N. Pleural SC5b-9 in differential diagnosis of tuberculous, malignant, and other effusions. Chest 1992; 102: 1060-1064.

36 Salomaa ER, Viander M, Saaresranta T, Terho EO. Complement components and their activation products in pleural fluid. Chest 1998; 114: 723-730.

37 Vives M, Porcel JM, Gázquez I, Pérez B, Rubio M. Pleural SC5b-9: a test for identifying complicated parapneumonic effusions. Respiration 2000; 67: 433-438.

38 Gaga M, Papamichalis G, Bakakos P, et al. Tuberculous effusion: ADA activity correlates with CD4+ cell numbers in the fluid and the pleura. Respiration 2005; 72: 160-165.

39 Losi M, Bossink A, Codecasa L, et al. Use of a T-cell interferon- $\gamma$ release assay for the diagnosis of tuberculous pleurisy. Eur Respir J 2007; 30: 1173-1179.

40 Kaisermann MC, Sardella IG, Trajman A, et al. IgA antibody responses to Mycobacterium tuberculosis recombinant MPT64 and MT-10.3 (Rv3019c) antigens in pleural fluid of patients with tuberculous pleurisy. Int J Tuberc Lung Dis 2005; 9: 461-466.

41 Kunter E, Cerrahoglu K, Ilvan A, et al. The value of pleural fluid anti-A60 IgM in BCG-vaccinated tuberculous pleurisy patients. Clin Microbiol Infect 2003; 9: 212-220.

42 Chierakul N, Damrongchokpipat P, Chaiprasert A, Arjratanakul W. Antibody detection for the diagnosis of tuberculous pleuritis. Int J Tuberc Lung Dis 2001; 5: 968-972.
43 Van Vooren JP, Farber CM, De Bruyn J, Yernault JC. Antimycobacterial antibodies in pleural effusions. Chest 1990; 97: 88-90.

44 Demkow U, Filewska M, Białas B, et al. [Antimycobacterial antibody level in pleural, pericardial and cerebrospinal fluid of patients with tuberculosis.]. Pneumonol Alergol Pol 2004; 72: 105-110.

45 Yokoyama T, Rikimaru T, Kinoshita T, Kamimura T, Oshita Y, Aizawa H. Clinical utility of lipoarabinomannan antibody in pleural fluid for the diagnosis of tuberculous pleurisy. J Infect Chemother 2005; 11: 81-83.

46 Morimoto T, Takanashi S, Hasegawa Y, et al. Level of antibodies against mycobacterial glycolipid in the effusion for diagnosis of tuberculous pleural effusion. Respir Med 2006; 100: 1775-1780.

47 Anie Y, Sumi S, Varghese P, Madhavi LG, Sathish M, Radhakrishnan VV. Diagnostic approaches in patients with tuberculous pleural effusion. Diagn Microbiol Infect Dis 2007; 59: 389-394.

48 Pai M, Flores LL, Hubbard A, Riley LW, Colford JM Jr. Nucleic acid amplification tests in the diagnosis of tuberculous pleuritis: a systematic review and metaanalysis. BMC Infect Dis 2004; 4: 6.

49 Ungerer JP, Oosthuizen HM, Retief JH, Bissbort SH. Significance of adenosine deaminase activity and its isoenzymes in tuberculous effusions. Chest 1994; 106: 33-37.

50 van Crevel R, Karyadi E, Netea MG, et al. Decreased plasma leptin concentrations in tuberculosis patients are associated with wasting and inflammation. J Clin Endocrinol Metab 2002; 87: 758-763.

51 Lagiou P, Signorello LB, Trichopoulos D, Tzonou A, Trichopoulou A, Mantzoros CS. Leptin in relation to prostate cancer and benign prostatic hyperplasia. Int $J$ Cancer 1998; 76: 25-28.

52 Hoheisel G, Izbicki G, Roth M, et al. Proinflammatory cytokine levels in patients with lung cancer and carcinomatous pleurisy. Respiration 1998; 65: 183-186.

53 Porcel JM, Vives M, Gázquez I, Vicente de Vera MC, Pérez B, Rubio M. Usefulness of pleural complement activation products in differentiating tuberculosis and malignant effusions. Int J Tuberc Lung Dis 2000; 4: 76-82.

54 Menzies D, Pai M, Comstock G. Meta-analysis: new tests for the diagnosis of latent tuberculosis infection: areas of uncertainty and recommendations for research. Ann Intern Med 2007; 146: 340-354.

55 Pai M, Riley LW, Colford JM Jr. Interferon- $\gamma$ assays in the immunodiagnosis of tuberculosis: a systematic review. Lancet Infect Dis 2004; 4: 761-776.

56 Steingart KR, Henry M, Laal S, et al. A systematic review of commercial serological antibody detection tests for the diagnosis of extrapulmonary tuberculosis. Thorax 2007; 62: 911-918.

57 Steingart KR, Henry M, Laal S, et al. Commercial serological antibody detection tests for the diagnosis of pulmonary tuberculosis: a systematic review. PLoS Med 2007; 4: e202.

58 Tiwari RP, Tiwari D, Garg SK, Chandra R, Bisen PS. Glycolipids of Mycobacterium tuberculosis strain H37Rv are potential serological markers for diagnosis of active tuberculosis. Clin Diagn Lab Immunol 2005; 12: 465-473. 
59 Pai M, Flores LL, Pai N, Hubbard A, Riley LW, Colford JM Jr. Diagnostic accuracy of nucleic acid amplification tests for tuberculous meningitis: a systematic review and meta-analysis. Lancet Infect Dis 2003; 3: 633-643.

60 Daley P, Thomas S, Pai M. Nucleic acid amplification tests for the diagnosis of tuberculous lymphadenitis: a systematic review. Int J Tuberc Lung Dis 2007; 11: 1166-1176.

61 Porcel JM, Vives M. Differentiating tuberculous from malignant pleural effusions: a scoring model. Med Sci Monit 2003; 9: CR175-CR180.

62 Villegas MV, Labrada LA, Saravia NG. Evaluation of polymerase chain reaction, adenosine deaminase and interferon- $\gamma$ in pleural fluid for the differential diagnosis of pleural tuberculosis. Chest 2000; 118: 1355-1364.

63 Neves DD, Dias RM, Cunha AJ. Predictive model for the diagnosis of tuberculous pleural effusion. Braz J Infect Dis 2007; 11: 83-88.

64 Burgess LJ, Maritz FJ, Le Roux I, Taljaard JJ. Combined use of pleural adenosine deaminase with lymphocyte/neutrophil ratio. Increased specificity for the diagnosis of tuberculous pleuritis. Chest 1996; 109: 414-419.

65 Trajman A, Kaisermann MC, Kritski AL, Sperhacke RD, Rossetti ML. Diagnosing pleural tuberculosis. Chest 2004; 125: 2366.

66 Pai M, Kalantri S, Dheda K. New tools and emerging technologies for the diagnosis of tuberculosis: part I. Latent tuberculosis. Expert Rev Mol Diagn 2006; 6: 413-422.

67 Pai M, Kalantri S, Dheda K. New tools and emerging technologies for the diagnosis of tuberculosis: part II. Active tuberculosis and drug resistance. Expert Rev Mol Diagn 2006; 6: 423-432.
68 Haykin S, ed. Neural Networks: a Comprehensive Foundation: International Edition. 2nd Edn. Harlow, Pearson Education, 1998.

69 Souza Filho JBO, Antunes PHS, Seixas JM, Maidantchik CLL. [Neural networks for the diagnosis of paucibacillary pulmonary tuberculosis.] In: Proceedings of the VIII Brazilian Conference on Neural Networks. Florianópolis, Brazilian Society for Neural Networks, 2007; pp. 1-5.

70 Maidantchik C, Seixas JM, Kritski A, et al. NEURALTB Web System. Support to the smear negative pulmonary tuberculosis diagnosis. In: Cardoso J, Cordeiro J, Filipe J, eds. ICEIS 2007 - Proceedings of the Ninth International Conference on Enterprise Information Systems, Volume AIDSS. Funchal, Institute for Systems and Technologies of Information, Control and Communication, 2007; pp. 198-203.

71 Bossuyt PM, Reitsma JB, Bruns DE, et al. Towards complete and accurate reporting of studies of diagnostic accuracy: the STARD initiative. Standards for Reporting of Diagnostic Accuracy. Clin Chem 2003; 49: 1-6.

72 Pai M, O'Brien R. Tuberculosis diagnostics trials: do they lack methodological rigor? Expert Rev Mol Diagn 2006; 6: 509-514.

73 Small PM, Perkins MD. More rigour needed in trials of new diagnostic agents for tuberculosis. Lancet 2000; 356: 1048-1049.

74 Dinnes J, Deeks J, Kunst H, et al. A systematic review of rapid diagnostic tests for the detection of tuberculosis infection. Health Technol Assess 2007; 11: 1-196.

75 Gardner MJ, Altman DG. Calculating confidence intervals for proportions and their differences. In: Gardner MJ, Altman DG, eds. Statistics with Confidence. 1st Edn. London, BMJ Books, 1989; pp. 28-33. 